\title{
Direct current cardioversion does not cause cardiac damage: evidence from cardiac troponin $\mathrm{T}$ estimation
}

\author{
A C R Rao, N Naeem, C John, P O Collinson, R Canepa-Anson, S P Joseph
}

\begin{abstract}
Aim-To determine whether elective direct current (dc) cardioversion of atrial fibrillation/flutter causes myocardial damage.

Methods and results-Cardiac troponin $\mathbf{T}$ and creatine kinase were estimated 20-28 hours after dc cardioversion in 51 patients who received dc shocks for elective cardioversion of chronic atrial fibrillation/ flutter. Although creatine kinase was raised in 44 patients, cardiac troponin $T$ was undetectable in all patients.

Conclusion-Cardiac damage does not occur as a result of cardioversion.

(Heart 1998;80:229-230)
\end{abstract}

Keywords: cardioversion; troponin $\mathrm{T}$; creatine kinase; atrial fibrillation

Direct current (dc) cardioversion ${ }^{1}$ revolutionised the management of cardiac arrhythmias. However, before the standardisation of outputs from external defibrillators there was controversy about the most appropriate strength of current to be used. ${ }^{2}$ Animal experiments have suggested that repeated high energy dc shocks result in myocardial damage. ${ }^{34}$ In humans, dc cardioversion causes a rise in the concentration of creatine kinase and its $\mathrm{MB}$ subfraction. ${ }^{5-7}$ This observation, along with the electrocardiogram (ECG) changes (repolarisation abnormalities) sometimes seen after dc cardioversion, led to the suggestion that dc cardioversion causes myocardial damage. Most of the creatine kinase released after cardioversion comes from the chest wall skeletal muscles, ${ }^{8}$ which also liberate the $\mathrm{MB}$ subfraction. Therefore, the extent that emergency dc cardioversion contributes to raised creatine kinase or its $\mathrm{MB}$ isoenzyme is unclear. The belief that myocardial damage might result from higher strengths of current may also have influenced recommendations about the choice of current strength. It remains uncertain whether dc cardioversion does actually cause myocardial damage.

Cardiac troponin $\mathrm{T}$ is a cardiac specific protein component of the troponin/tropomyosin complex. ${ }^{9}$ A rise in serum troponin $\mathrm{T}$ concentrations is specific for cardiac damage. It remains raised for up to 48 hours after myocardial injury, thus providing a wide time window. ${ }^{9}$ Currently available data suggest that the kinetics of cardiac troponin $\mathrm{T}$ release from acutely damaged myocardium are identical whatever the cause of damage. ${ }^{10}$
Our study was undertaken to determine whether there was any evidence of myocardial damage after elective dc cardioversion of atrial fibrillation/flutter by measuring concentrations of cardiac troponin $\mathrm{T}$ in patients undergoing $\mathrm{dc}$ cardioversion.

\section{Methods}

Fifty one consecutive patients undergoing elective dc cardioversion for atrial fibrillation/ flutter were entered into the study. None had chronic renal failure, acute myocardial infarction, or skeletal muscle disease. As described previously, ${ }^{11} \mathrm{dc}$ shocks were administered in increasing strengths of $100 \mathrm{~J}, 200 \mathrm{~J}, 300 \mathrm{~J}$, and $360 \mathrm{~J}$ (only one shock of each strength), until either sinus rhythm was restored or $360 \mathrm{~J}$ was applied.

A blood sample was taken 20-28 hours after dc cardioversion. Samples were centrifuged and analysed or frozen at $-70^{\circ} \mathrm{C}$ until analysis (all analyses were performed within two weeks). Cardiac troponin $\mathrm{T}$ was estimated by enzyme linked immunosorbent assay as described previously. ${ }^{9}$ The relation between shock strength and the myocardial marker was determined by the Spearman rank correlation coefficient.

\section{Results}

Of the 51 patients, 40 were men. The median age was 66 years (range, 40-83; lower quartile, 61; upper quartile, 69). Twenty eight received a total current strength of $960 \mathrm{~J}$, six received $600 \mathrm{~J}$, one received $400 \mathrm{~J}, 11$ received $300 \mathrm{~J}$, and five received $100 \mathrm{~J}$ (median, $960 \mathrm{~J}$; lower quartile, $100 \mathrm{~J}$; upper quartile, $960 \mathrm{~J}$ ). Sinus rhythm was restored in 33 patients. Cardiac troponin $\mathrm{T}$ was not detected in any of these patients (lower limit of the troponin $\mathrm{T}$ assay is $0.05 \mu \mathrm{g} / \mathrm{l})$ but creatine kinase was raised in 44 patients. There was a strong correlation between cumulative current strength and the serum concentration of creatine kinase (fig 1). The Spearman rank correlation coefficient $r s$ was 0.7347 (95\% confidence intervals (CI) 0.5757 to $0.8402 ; \mathrm{p}<0.0001)$.

\section{Discussion}

Animal experiments have demonstrated both histological and biochemical evidence of myocardial damage after dc cardioversion. ${ }^{3}{ }^{4}$ However, in these studies, large strengths of current were used repeatedly. In humans, the strength of current delivered by the defibrillator has been standardised, and is considerably lower than the strengths used in animal experiments. 


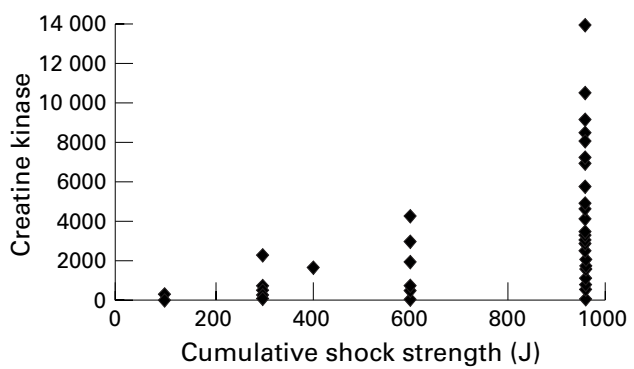

Figure 1 Cumulative shock strength and creatine kinase concentration.

Studies in man have consistently demonstrated raised creatine kinase concentrations after dc cardioversion. ${ }^{5-7}$ Most of the creatine kinase is released from the pectoral muscles. ${ }^{8}$ The MB subfraction of creatine kinase is raised in a smaller number of patients. This finding, along with the observation that post-dc cardioversion ECGs demonstrate repolarisation changes in some patients, ${ }^{12}$ has led to the belief that myocardial damage might occur after dc cardioversion. Small amounts of the MB subfraction are also released from the skeletal muscle. Therefore, the specificity of creatine kinase and its MB subfraction in the detection of myocardial damage after dc cardioversion is limited, as is their value for the diagnosis of acute myocardial infarction in patients who receive dc cardioversion at or soon after admission.

Cardiac troponin $\mathrm{T}$ has emerged as a marker of high sensitivity and specificity for the detection of myocardial damage. ${ }^{9}$ Previous experience in patients with acute myocardial infarction has shown that it is raised for up to 48 hours after initial myocardial injury. ${ }^{9}$ This provides a large time window for detection. Currently available data suggest that the kinetics of cardiac troponin $T$ release from acutely damaged myocardium are identical whatever the cause of damage. ${ }^{10} \mathrm{~A}$ rise in cardiac troponin $\mathrm{T}$ does not result from skeletal muscle damage. ${ }^{13} 14$ In the present study, cardiac troponin $\mathrm{T}$ was not detected after dc cardioversion in patients receiving a standard strength of current. Therefore, myocardial damage does not result from elective dc cardioversion. It is likely, therefore, that any rise in cardiac troponin $\mathrm{T}$ seen after dc cardioversion is a result of myocardial injury unrelated to the cardioversion.
Similar results have been obtained in a recent study using cardiac troponin $\mathrm{I},{ }^{15}$ which, like cardiac troponin $T$, is a cardiac specific marker of equally high sensitivity and specificity. ${ }^{16}$

In our study, more than half of the patients were successfully cardioverted. Success of dc cardioversion of atrial fibrillation is highly variable $^{11}$ and is usually related to patient selection. Some have suggested that the use of anteroposterior electrodes and a second shock at $360 \mathrm{~J}$ might be useful for improving success rates. ${ }^{17}$

In conclusion, dc cardioversion using standard current strengths does not cause myocardial damage.

1 Lown B, Amarasingham R, Neuman J. New method for terminating cardiac arrhythmias: use of synchronised capacitor discharge. $7 A M A 1962 ; 182: 548-55$.

2 Lown B, Crampton RS, DeSilva RA, et al. The energy for ventricular defibrillation-too little or too much? $N$ Engl f Med 1978;298:1252-3.

3 Wilson CM, Allen JD, Bridges JB, et al. Death and damage caused by multiple direct current shocks: studies in an animal model. Eur Heart f 1988;9:1257-65.

4 Trouton TG, Allen JD, Yong LK, et al. Metabolic changes and mitochondrial dysfunction early following transthoracic countershocks in dogs. PACE 1989;12:1827-34.

5 Reiffel JA, Gambino SR, McCarthy DM, et al. Direct current cardioversion. Effect on creatine kinase, lactic dehydrogenase and myocardial isoenzymes. $\mathcal{F A M A} 1978$; 239:122-4.

6 Jackobsson J, Odmansson I, Nordlander R. Enzyme release after cardioversion. Eur Heart f 1990;11:749-52.

7 Ehsani A, Ewy GA, Sobel BE. Effects of electrical countershock on serum creatinine phosphokinase (CPK) isoenzyme activity. Am $\mathcal{f}$ Cardiol 1976;37:12-18.

8 Konttinen A, Hulpl, Louhlja A, et al. Origin of elevated serum enzyme activity after direct current countershock. $N$ Engl f Med 1969;281:231-4.

9 Collinson PO, Mosley D, Stubbs PJ, et al. Troponin T for the differential diagnosis of ischaemic myocardial damage. Ann Clin Biochem 1993;30:11-16.

10 Mair J, Diensti F, Puschendorf B. Cardiac troponin T in the diagnosis of myocardial injury. Crit Rev Clin Lab Sci 1992; 29:31-57.

$11 \mathrm{Lip} \mathrm{GYH}$. Cardioversion of atrial fibrillation. Postgrad Med $\mathcal{F}$ 1995;71:457-65.

12 Van Gelder IC, Crijns HJ, Van Der Laarse A, et al. Incidence and clinical significance of ST segment elevation after electrical cardioversion of atrial fibrillation and flutter. $\mathrm{Am}$ Heart $\mathcal{F}$ 1991;121:51-6.

13 Collinson PO, Chandler HA, Stubbs PJ, et al. Cardiac troponin $\mathrm{T}$ and $\mathrm{CK}-\mathrm{MB}$ concentration in the differential diagnosis of elevated creatine kinase following arduous physical training. Ann Clin Biochem 1995;32:450-3.

14 Mair J, Wohlfarter T, Koller A, et al. Serum troponin T after extraordinary endurance exercise [letter]. Lancet 1992;340: 1048 .

15 Bonnefoy E, Chevalier P, Kirkorian G, et al. Cardiac troponin I does not increase after cardioversion. Chest 1997;111:15-18.

16 Collinson PO. To T or not to T, that is the question [editorial]. Clin Chem 1997;43:421-3.

17 Walters MI, Norell MS. Energy considerations for the electrical conversion of atrial fibrillation. $B r f$ Cardiol 1997;4:177-8. 